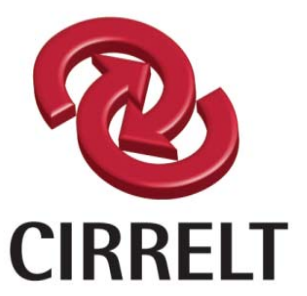

Centre interuniversitaire de recherche sur les réseaux d'entreprise, la logistique et le transport

Interuniversity Research Centre on Enterprise Networks, Logistics and Transportation

\title{
Designing the Master Schedule for Demand-Adaptive Transit Systems
}

\author{
Teodor Gabriel Crainic \\ Fausto Errico \\ Federico Malucelli \\ Maddalena Nonato
}

May 2008

CIRRELT-2008-18

Bureaux de Montréal: Université de Montréal C.P. 6128, succ. Centre-ville Montréal (Québec)

Canada $\mathrm{H} 3 \mathrm{C} 3 \mathrm{~J} 7$

Téléphone: 514 343-7575

Télécopie: 514 343-7121
Bureaux de Québec:

Université Laval

Pavillon Palasis-Prince, local 2642

Québec (Québec)

Canada G1K 7P4

Téléphone : 418 656-2073

Télécopie : 418 656-2624

www.cirrelt.ca 


\title{
Designing the Master Schedule for Demand-Adaptive Transit Systems
}

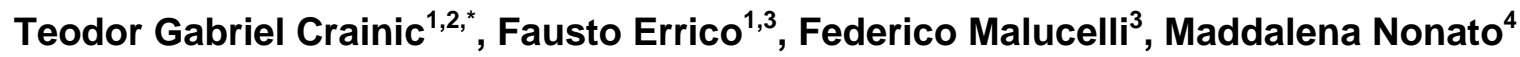 \\ ${ }^{1}$ Interuniversity Research Centre on Enterprise Networks, Logistics and Transportation (CIRRELT) \\ 2 Department of Management and Technology, Université du Québec à Montréal, C.P. 8888, \\ succursale Centre-ville, Montréal, Canada H3C 3P8 \\ 3 Politecnico di Milano, DEI, Piazza Leonardo da Vinci, Milano, Italy, 20133 \\ ${ }^{4}$ University of Ferrara, via Saragat 1, Ferrara 44100, Italy
}

\begin{abstract}
Demand-Adaptive Systems (DAS) display features of both traditional fixed-line bus services and purely on-demand systems such as dial-a-ride, that is they offer demand-responsive services within the framework of traditional scheduled bus transportation. A DAS bus line serves, on one hand, a given a set of compulsory stops according to a predefined schedule specifying the time windows associated to each, providing the traditional use of the transit line, without requiring any reservation. On the other hand, passengers may also issue requests for transportation between two desired, optional, stops, which induces detours in the vehicle routes. The design of a DAS line is a complex planning operation that requires to determine not only its design in terms of selecting the compulsory stops, but also its master schedule in terms of the time windows associated to the compulsory stops. Designing a DAS thus combines elements of strategic and tactical planning. In this paper we focus on determining a master-schedule for a single DAS line. We propose a mathematical description and a solution framework based on the estimation of a number of statistical parameters of the demand and the DAS line service. Results of numerical experiments are also given and analyzed. Cooperation as problem-solving and algorithm-design strategy is widely used to build methods addressing complex discrete optimization problems. In most cooperative-search algorithms, the explicit cooperation scheme yields a dynamic process not deliberately controlled by the algorithm design but inflecting the global behaviour of the cooperative solution strategy. The paper presents an overview of explicit cooperation mechanisms and describes issues related to the associated dynamic processes and the emergent computation they often generate. It also identifies a number of research directions into cooperation mechanisms, strategies for dynamic learning, automatic guidance, and selfadjustment, and the associated emergent computation processes.
\end{abstract}

Keywords. Public transit, demand-responsive systems, demand-adaptive systems, scheduling.

Acknowledgements. Partial funding for this research was provided by Natural Sciences and Engineering Research Council of Canada (NSERC) through its Industrial Research Chair and Discovery grant programs. This research was also partially funded by Regione Lombardia, Italy, through its INGENIO grants. Fausto Errico wishes to thank Professor Stein W. Wallace for an enlightening conversation held in Montreal in December 2006.

Results and views expressed in this publication are the sole responsibility of the authors and do not necessarily reflect those of CIRRELT.

Les résultats et opinions contenus dans cette publication ne reflètent pas nécessairement la position du CIRRELT et n'engagent pas sa responsabilité.

* Corresponding author: Teodor-Gabriel.Crainic@cirrelt.ca

Dépôt légal - Bibliothèque nationale du Québec, Bibliothèque nationale du Canada, 2008

(c) Copyright Crainic, Errico, Malucelli, Nonato and CIRRELT, 2008 


\section{Introduction}

Traditional transit services are particularly suited to handle situations where the demand for transportation is strong, i.e., when there is a consistently high demand over the territory and for the time period considered. The high degree of resource sharing by a large number of passengers makes it then possible to provide efficiently and economically high quality, i.e., frequent, services operating generally high-capacity vehicles over fixed routes and schedules. Routes and schedules may and do vary during the day, but, in almost all cases, they are not dynamically adjusted to the fluctuations of demand. In contrast, when the demand for transportation is weak, e.g., during out of rush-hour periods or in low-population density zones, operating a good-quality traditional transit system is very costly. In particular the fixed structure of traditional transit services cannot economically and adequately respond to significant variations in demand.

Demand-responsive systems are a family of mass transportation services which, as their name suggests, are responsive to the actual demand for transportation in a specific time period. Such services evolve toward a personalization of mass services: itineraries, schedules, and stop locations are variable and determined according to the needs for transportation as they change in time. Demand-responsive systems were introduced under the name of Diala-Ride $(D A R)$ as door-to-door services for users with particular needs or reduced mobility, such as handicapped and elderly people $[12,8]$. The flexibility of DAR systems to respond to varying individual requests for transportation provides the means to offer more personalized services, while still maintaining a certain degree of resource sharing. This has lead certain transportation or city authorities to extend DAR services to more general transportation settings.

DAR systems display, however, a number of drawbacks, some of which follow from the extreme flexibility inherent in the system definition. Thus, for example, because the supply of transportation service changes according to needs expressed for particular time periods, neither the transit operator nor the users may predict the vehicle itineraries, stop locations, and associated schedules. As a consequence, users are obliged to book the service well in advance of the actual desired time of utilization and the actual pick up time is very much left to the discretion of the operator. For similar reasons, it is extremely difficult to integrate DAR and other traditional transit services.

A new type of demand-responsive systems, denoted Demand-Adaptive System (DAS) has been introduced to address some of these issues [10,11]. DASs are transit services displaying features of both traditional fixed-line bus service and purely on-demand systems such as DAR. In other words, a DAS attempts to offer demand-responsive services within the framework of traditional scheduled bus transportation. A DAS bus line serves, on one hand, a given a set of compulsory stops according to a predefined schedule specifying the time windows associated to each, providing the traditional use of the transit line, without in-advance reservations. On the other hand, similarly to DAR services, passengers may issue requests for transportation between two desired, optional stops (not necessarily on the same 
line), which induces detours in the vehicle routes.

Similarly to most transportation systems dedicated to serve several demands with the same vehicle, traditional transit systems involve a complex planning system made up of many interrelated decisions. Schematically, the design of the system in terms of line routes is determined during the so-called strategic planning phase, timetables and vehicle schedules and routes are part of the tactical planning phase, and crew schedules are built during operational planning (Ceder et al. [2]). Comparatively, purely on-demand services such as DAR, need little strategic design, mainly to define service areas and the composition of the fleet (e.g., number and type of vehicles). The most important planning process for DAR is at the operational level when routes and schedules are determined little time before actual operations and are possibly dynamically modified one service has begun.

DAS services combining characteristics of traditional and on-demand systems require both a system-design phase and an operational, time and user request-dependent adjustment of vehicles routes and schedules. The latter has been addressed in Crainic et al. [6] and Malucelli et al. [9]. The former forms the topic of this paper. It is, in a certain sense, a more complex planning operation than for traditional transit because it requires not only to determine the design of the line as the selection of the compulsory and optional stops, but also the determination of the time windows associated to the compulsory stops. Designing a DAS thus combines elements of strategic and tactical planning. To emphasize this characteristics, we identify the output of the process and the master-route network and the master-schedule, respectively.

In this paper we focus on determining a master-schedule for a single DAS line. We propose a mathematical description and a solution framework based on the estimation of a number of statistical parameters of the demand and the DAS line service. A sampling approach is used for the estimations.

The remaining part of the paper is organized as follows. We give a brief description of DAS services in Section 2, while commonalities and differences among scheduling DAS, DAR, and traditional transit services are discussed in Section 3. Section 4 is dedicated to the description of the DAS line master-scheduling problem and the solution framework we propose. We discuss the effectiveness of the method end computational results in Section 5 and conclude in Section 6.

\section{Demand Adaptive Systems}

Demand adaptive systems were first introduced by Malucelli, Nonato, and Pallottino [10] and then treated in a more general context by Crainic et al. [6] (see also, [5, 4, 9]). A similar type of service is also described in [11]. 
A DAS targets low-density/volume demand areas and attempts to conjugate the advantages of traditional transit transportation services and the flexibility of on-demand personalized services. It is based on the observation that even in such areas there are locations where a relatively important part of the overall demand may be consistently found: railway and underground stations, shopping centers, hospitals, etc. This leads to the possibility to economically design a backbone transit service covering these most attractive stops, while allowing vehicles to detour as needed to pick up and drop off passengers at the other stops. The latter capability, combined to an on-request booking system, increases customer satisfaction and the dimension of the potential user group.

In its most general form, a DAS is made up of several lines and is connected to the lines of the traditional transit system. Several vehicles operate on each DAS line providing service among a sequence of compulsory stops. Each compulsory stop is served within a predefined time window. The collection of time windows corresponding to the compulsory stops, including the start and end of the line, makes up the master schedule of the DAS line. This makes up the traditional part of a DAS. Additional service and flexibility is provided by allowing customers to request service from and to optional stops, that is, stops which are served only if a request is issued and it is accepted. We identify users which request service at an optional stop as active, while users moving only between compulsory stops are identified as passive.

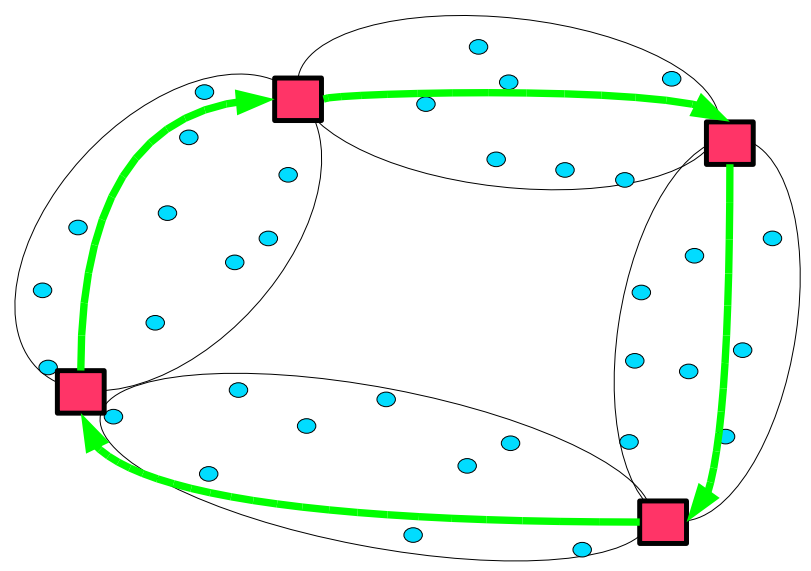

Figure 1: A Basic DAS Line Serving the Compulsory Stops

To serve optional stops, the vehicle must generally deviate from the shortest path joining two successive compulsory stops. The set of optional stops that it is possible to visit between two consecutive compulsory stops is part of the design of the DAS line and is denoted segment. An optional stop cannot belong to more than one segment. Figure 1 depicts the basic DAS service of the compulsory stops, while Figure 2 illustrates the same DAS line when user requests for optional stops are present.

Transfers between DAS lines and between these and regular transit lines take place at compulsory stops. Time windows play an important role in this context because they establish time relations among different DAS and traditional lines which share the same com- 


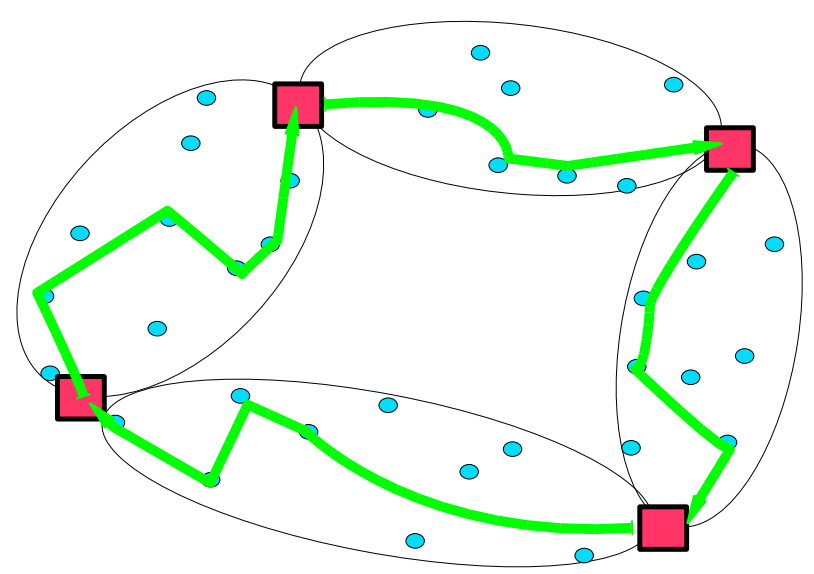

Figure 2: The DAS Line Serving Optional Stops

pulsory stops. The time windows in the master schedule also influence the flexibility the service may provide for user requests at optional stops. The wider they are, the more flexibility there is available. Yet, it is not possible to increase their width arbitrarily, because the service would slow down excessively, loosing attractiveness. Notice finally, that the time windows and the segment specification provide an a priori guarantee relative to the longest time users might have to spend traveling on the line. In any case, the detours associated to optional stops must be consistent with the time windows at compulsory stops.

\section{Scheduling Issues in DAS, DAR and Traditional Tran- sit Systems}

Scheduling is a fundamental planning activity for any transportation system, in particular for DASs, DAR and traditional transit systems. The nature of the scheduling process changes significantly, however, according to the type of service at hand.

In traditional transit systems, a schedule indicates the passing times at each stop of each line. Vehicles are supposed to follow these times as strictly as possible, since users of the system base their trip plans on the published schedules. Scheduling problems in traditional transit system belong to the so-called tactical planning level, the line definitions and the service frequencies being usually assumed known. Once the schedule has been established, it remains unchanged for medium-term periods, such as six months or one year. For a more in-depth discussion of scheduling issues in traditional transit systems, the interested readers are referred to $[2]$.

The situation is different for DAR systems. Schedules are still indicating vehicle itineraries, stops, and passing times, but these particular to each vehicle tour according to the actual requests for transportation accepted for the corresponding time period. This corresponds 
to an operational planning level activity that decides on all schedule components (i.e., line itinerary, stops, and passing times), which are valid only for the duration of the specific service. See [3] for a review of the topic.

The case of DASs is more complex. Because DAS aims to provide demand-responsive services within the framework of traditional scheduled transit transportation, its scheduling combines the two planning processes briefly sketched above. Two schedules are thus built. A master schedule defines the partial line (vehicle) itineraries, the sequence of compulsory stops, and time windows at these stops. This schedule plays the same role for the transit authority and the passive users of the system as the schedule in traditional transit systems. At operation time, the actual schedule is built to incorporate the additional, optional stops corresponding to the accepted active-user requests, while respecting the time windows constraints imposed by the master schedule. The problem of finding a DAS schedule at operational level was addressed in $[9,6]$.

Building the master schedule is a tactical planning level activity, where actual service times at compulsory stops are modified according to the season. It is also an important component of the strategic planning process, as the definition of the segments making up the line requires the specification of time windows at compulsory stops. The next section is dedicated to the these issues for a single DAS line.

\section{The DAS Line Master Scheduling Problem}

This section is dedicated to the issue of determining the master schedule of a single DAS line, that is, determining the time windows for the compulsory stops of the line. This so-called DAS Line Master Scheduling Problem (DLMSP) may be viewed as the last stage of the DAS line design problem which is addressed in more depth in [7].

The single-line DAS design problem assumes that the territory to be covered by the DAS line has been determined, the travel times between any pair of potential stops in the territory (these include transfer points to other lines or transportation systems) have been accurately estimated, and that a measure of the transportation demand among the potential stops is available. For a given time horizon where demand is assumed stable (e.g., morning rush hour), the DAS line design problem is made up of several interrelated decisions regarding the selection of compulsory stops among all the potential stops in the territory, their sequencing, the partitioning of the optional stops into segments, and the determination of the master schedule, that is the definition of the time windows vehicles will have to respect at compulsory stops. The first three components make up the so-called topological-design phase of the problem and a number of methodological approaches are proposed in [7] to address various problem settings, e.g., objectives to be satisfied (vehicle cost, travel time experienced by users, a combination thereof, etc. - we use a combination), whether all potential stops should be reachable by the designed line, and so on. The last component of 
the design process constitutes the object of this paper.

A formal model for DLMSP is presented in Section 4.1. In Section 4.2 we focus on the Single Segment Master Scheduling Problem, a core subproblem in addressing the DLMSP. Section 4.3 presents the complete solution approach we propose for the DLMSP.

\subsection{Problem description and modeling}

The problem of building the DAS line master schedule, that is, to fix the time windows at all compulsory stops of the line, assumes two inputs. The first consists in the topological design of the line: the ordered set of compulsory stops and the associated set of optional stops partitioned into segments. The demand for transportation between the stops of the line makes up the second input.

The choice of time windows must be performed taking into account several conflicting goals. First, the master schedule should provide sufficient time between compulsory stops such that, during actual operations, vehicles may serve all requests for service at optional stops. Second, for economical reasons, the total maximum time of the line, travel and stops, to be as short as possible. Finally, quality of service criteria also induce conflicting actions: while users already on the bus prefer narrow time windows, to avoid long delays at compulsory stops, and short travel times between consecutive compulsory stops, to avoid being on the bus for long, users at optional stops prefer longer travel times that allow vehicles to detour by their stop.

To illustrate the incompatibility of these goals, consider that simultaneously enforcing small time windows and high probability of being able to serve all potential requests implies a rather long travel time for each segment compared to the corresponding shortest path. But, since the actual number of requests is usually small compared to the total potential number, such a strategy would result in vehicles arriving at compulsory stops well before the earliest departure time, significant dead times at compulsory stops for users, and long ride times for the line. To avoid this, time windows have to be smaller, resulting in a smaller probability of being able to serve the whole set of issued requests. This is indeed general DAS operational policy (Malucelli, Nonato, and Pallottino [10] and Crainic et al. [6]), requests that cannot be accommodated being either lost, served by a later vehicle or by taxi, according to the policy of the transit company.

We thus assume a maximum width for time windows at compulsory stops and aim to select their actual widths to minimize the maximum vehicle ride time, while guaranteeing to serve the set of requests with a given probability. The maximum width and the service probability are, of course, managerial decisions and thus application dependent.

Demand for transportation is usually described as the number of potential trips that might be requested during the time period considered for each pair of stops. Based on this 
information, it is straightforward to compute the probability of at least a request being issued for a given pair of stops, as well as the probability of each optional stop of being requested for service either to board or to alight a vehicle. We work with this last set of probabilities in the model we propose. Consequently, the goal of serving the whole set of requests with a given probability becomes serving the whole set of requested stops with a given probability. This makes it easier to address the problem.

To formally write the model, consider a sequence of compulsory stops $H=\left\{f_{0}, f_{1}, \ldots, f_{n}\right\}$. Sets of optional stops $F_{h}$, with $h=1, \ldots, n$ are associated with each pair of consecutive compulsory stops $\left\langle f_{h-1}, f_{h}\right\rangle$. The sets $F_{h}$ are mutually disjoint. We define a directed graph $G_{h}=\left(N_{h}, A_{h}\right)$ for any pair $\left\langle f_{h}, f_{h+1}\right\rangle$ such that $N_{h}=F_{h} \cup\left\{f_{h-1}, f_{h}\right\}$ and $A_{h}=N_{h} \times N_{h}$. We call $G_{h}$ a segment and $G=\cup G_{h}$. A traversing (travel) time $c_{i j}$ is associated to each arc $(i, j) \in A$. A probability $p_{i}$ of being requested for service is associated to each optional stop $s_{i} \in \cup_{h} F_{h}$.

The DLMSP consists in associating to each compulsory stop $f_{h}$ a time window $\left[a_{h}, b_{h}\right]$ such that the vehicle must not leave the compulsory stop $f_{h}$ before $a_{h}$, nor after $b_{h}$; it is allowed, however, to arrive to $f_{h}$ before $a_{h}$. Our goal is to find the best sequence $\left\{\left\langle a_{1}, b_{1}\right\rangle,\left\langle a_{2}, b_{2}\right\rangle, \ldots,\left\langle a_{n}, b_{n}\right\rangle\right\}$ which, with a given probability $P_{\epsilon}$, guarantees service for $e v$ ery optional stop which can be requested for service. We define the best sequence as the one displaying the smallest value of $b_{n}$.

For the sake of simplicity of exposition, we consider the case where all time windows are of equal width $b_{h}-a_{h}=\delta$, which reduces the problem to that of finding the best sequence $\left\{b_{0}, b_{1}, \ldots, b_{n}\right\}$. The procedure we propose extends straightforwardly, however, to the cases when 1) the time windows are fixed but different for the compulsory stops, and 2) time windows are bounded by a maximum width value but are allowed to be smaller. The latter case could also consider finding, for a given compulsory stop $f_{h}$, the maximum $a_{h}$ which guarantees with a given probability no vehicle dead time at $f_{h}$.

We now focus on a core subproblem that estimates the travel time, and thus the time window at the destination compulsory stop, of a single segment. The full algorithm will then bring together the sequence of segments making up the route.

\subsection{The Single Segment Subproblem}

How long does it take to travel a segment? The answer obviously depends on what optional stops have to be serviced and this is usually different each time a vehicle travels the line and the segment, because service at optional stops follows particular user requests that have been accepted. Consequently, such operational information must be estimated for the tacticalplanning purpose of building a master schedule. We propose a statistical estimation of the travel time of a given segment based on an efficient sampling procedure. 
Consider the generic segment $G_{h}=\left(N_{h}, A_{h}\right)$, and recall that $N_{h}=F_{h} \cup\left\{f_{h-1}, f_{h}\right\} f_{h-1}$ and $f_{h}$ are the initial and terminal compulsory stops of the segment, respectively, $F_{h}$ is a set of optional stops, and $A_{h}=N_{h} \times N_{h}$. Let $\bar{L}_{h-1}$ represent the departure time from compulsory stop $f_{h-1}$.

As indicated previously, each optional stop $s_{i} \in F_{h}$ has a positive known probability $p_{i}$ of being active, that is, of being requested for service during a particular vehicle run. We assume these probabilities to be mutually independent. The set of optional stops that are simultaneously active during a vehicle run, $S_{h} \subseteq N_{h}$, is denoted the active set (with every $s_{i} \in S_{h}$ being active, while every $s_{i} \in N_{h} \backslash S_{h}$ not being active). The probability of any set $S_{h}$ to be active, $p_{S_{h}}$, is positive and may be easily derived from the probabilities $p_{i}$ of its active optional stops.

The time required by a vehicle to travel from the initial to the terminal compulsory stop of segment $h$ serving a given set $S_{h}$ of active optional stops in $S_{h}$ is denoted $H_{h}$ and is called the service time of set $S_{h}$. Assuming an efficient operation of the line, the service time $H_{h}$ may be approximated at planning level as the duration of the shortest path starting in $f_{h-1}$, ending in $f_{h}$, and passing by all the stops of the active set $S_{h}$. We thus used a Minimum Hamiltonian Path solver to compute $H_{h}$ for our experimentations. (More sophisticate procedures may be implemented to take advantage of particular application attributes, but this does not change the general behavior of the proposed methodology.)

The service time associated to segment $h$ is of course a random variable at planning level and we denote it $H_{h}(\omega)$. The goal of the Single Segment Meta Schedule Problem (SSMST) is to determine the lowest value $b_{h}$ which guarantees, with probability $1-\epsilon$, that the vehicle has sufficient time to serve an active set. That is, fix $b_{h}=H_{h}^{1-\epsilon}+\bar{L}_{h-1}$, where $H_{h}^{1-\epsilon}$ is such that $\mathcal{P}\left\{H_{h}(w) \leq H_{h}^{1-\epsilon}\right\} \geq 1-\epsilon$.

The computation of $H_{h}^{1-\epsilon}$ requires the knowledge of the Cumulative Distribution Function (CDF) and thus of the Probability Mass Function (PMF) of the random variable $H_{h}(\omega)$. Since the latter requires $2^{\left|N_{h}\right|}$ Minimum Hamiltonian Path computations, this approach is not computationally affordable in most cases. Consequently, we estimate the PMF and sampling appears as the method of choice.

It is difficult to estimate how large a sample that represents adequately the population of active sets should be, but we suppose it could become quite large. Then, for computing efficiency reasons, we propose instead the very simple following algorithm:

- Take a number $\left\{r_{1}, r_{2}, \ldots, r_{l}\right\}$ of random samples of relatively small cardinality;

- For each sample $r_{k}$, compute its $P M F_{k}$ and $C D F_{k}$, as well as the value of $b_{h}^{k}$;

- Compute the mean value and standard deviation of $b_{h}^{k}$; If the standard deviation is close to the mean value, that is if the solution is precise, stop;

- Otherwise, increment the cardinality of the samples and iterate the previous steps. 
The undeniable advantage of this algorithm is its simplicity. On the other side, one cannot guarantee an unbiased solution, nor that the dimension of the samples will stay within computationally reasonable dimensions. A number of parameters (e.g., the number of samples) must also be calibrated. Yet, as illustrated by the results of Section 5, the method is very effective and adverse effects are not noticeable.

\subsection{Solution Approach to DLMSP}

We now present the complete solution method for the DAS line master schedule problem, where we need to sew segments together.

In the previous subsection, we decoupled segments by assuming that the vehicle departure time from its initial compulsory stop $f_{h-1}$ was known for each segment $G_{h}$. Actually, this is true for the first segment only, the departure time from the first compulsory stop $f_{0}$ being here arbitrarily denoted $t=0$, which also translates into $\mathcal{P}\left\{L_{0}(\omega)=0\right\}=1$. For all subsequent segments, the departure time depends upon the arrival time of the previous segment, which depends on its departure and service times, which depends upon ... Since service times are random variables, segment departure times for all segments but the first are also random variables.

We therefore introduce the random variable $L_{h}(\omega)$ representing the vehicle departure time from compulsory stop $f_{h}$, where $a_{h} \leq L_{h}(\omega) \leq b_{h}$. We introduce also the random variable $T_{h}(\omega)$ :

$$
T_{h}(\omega)=L_{h-1}(\omega)+H_{h}(\omega) .
$$

not constrained to belong to $\left[a_{h}, b_{h}\right]$. The vehicle departure times at two consecutive compulsory stops and the service time for the segment to which they belong to are then related as follows:

$$
L_{h}(\omega)= \begin{cases}T_{h}(\omega) & \text { if } \omega \mid a_{h} \leq T_{h}(\omega) \leq b_{h} \\ a_{h} & \text { if } \omega \mid T_{h}(\omega)<a_{h} \\ b_{h} & \text { if } \omega \mid T_{h}(\omega)>b_{h} .\end{cases}
$$

We assume in the following, without loss of generality but with a simplified notation, that as long as a vehicle arrives at a compulsory stop $f_{h}$ during the interval $\left[a_{h}, b_{h}\right]$, the arrival and departure times coincide.

Recall that the value $b_{h}$ for segment $G_{h}$ has to be such that it is possible to serve all possible active sets with a given probability. We must therefore compute the PMF (and consequently of the CDF) of $T_{h}(\omega)$, that is, select $b_{h}=T_{h}^{1-\epsilon}$, where $T_{h}^{1-\epsilon}$ is such that $P\left\{T_{h}(\omega) \leq T_{h}^{1-\epsilon}\right\}>1-\epsilon$.

Notice that, by hypothesis, $H_{h}(\omega)$ and $L_{h-1}(\omega)$ are independent. Consequently, the PMF of their summation, $T_{h}(\omega)$, can be computed through the simple convolution of the PMFs of $H_{h}(\omega)$ and $L_{h-1}(\omega)$. The problem of finding $b_{h}$ then reduces to the problem of estimating 
the PMF of $H_{h}(\omega)$ and $L_{h-1}(\omega)$. We showed in the previous subsection how to compute the CDF of $H_{h}(\omega)$. The CDF of $L_{h-1}(\omega)$ may be easily obtained from that of $T_{h-1}(\omega)$.

We can now present the scheme of the algorithm we propose for the DLMSP. The algorithm accepts as input the sequence of segments $G=\cup_{1,2, \ldots, n} G_{h}$ and the service probability $P_{\epsilon}=(1-\epsilon)^{n}$, and proceeds as follows:

1. For every segment $G_{h}, h \in\{1,2, \ldots, n\}$

(a) Compute PMF and CDF of $L_{h}(\omega)$

(b) Compute PMF and CDF of $H_{h}(\omega)$

(c) Compute PMF and CDF of $T_{h}(\omega)$ as the convolution of the PMFs of $L_{h-1}$ and $H_{h}$

(d) Compute $T_{h}^{1-\epsilon}$ and set $b_{h}=T_{h}^{1-\epsilon}$.

The algorithm returns the best sequence $\left\{b_{1}, b_{2}, \ldots, b_{n}\right\}$ of latest departure times for the segments, such that any randomly requested optional stop is served with probability $P_{\epsilon}=$ $(1-\epsilon)^{n}$.

\section{Results}

This section is dedicated to a discussion of computational results relative to the estimation of the PMF of random variables $H_{h}(w)$ when considering decoupled segments, as well as of the corresponding values $H_{h}^{1-\epsilon}$. This is in fact the core point of the solution methodology proposed. Experimental results support, in particular, the claim that precise and unbiased values of $H_{h}^{1-\epsilon}$ are obtained even for relatively small sample dimensions.

We tested our algorithm over square-shaped segments with initial and terminal compulsory stops located at the extremities of one of the diagonals. Optional stops are uniformly distributed on the square. Distances between optional stops are Euclidean and traveling times are proportional to distances with proportionality constant 1 . We generated instances with a number of stops, including the two compulsory ones, varying from 20 to 50 by steps of 10 . To each optional stop is associated a probability included in the open interval $(0,1)$. For each problem dimension we randomly generated three instances, different both in the possible locations of optional stops and the probabilities associated to them.

Table 1 displays the main features of the test problem instances: name, time length of the longest path with only one optional stop, the time length of the Hamiltonian path, the sum of the probabilities associated to optional stops, and the number of optional stop with probability grater than $50 \%$. Hamiltonian paths are computed with a modified version of the Asymmetric TSP code available in [1]. 


\begin{tabular}{|c|c|c|c|c|}
\hline Name & Longest 1-Path & Hamiltonian Path & Sum Prob & $>50 \%$ \\
\hline A20 & 1924.8 & 4113 & 9.03 & 9 \\
\hline B20 & 1925.43 & 4032 & 9.31 & 9 \\
\hline C20 & 1634.38 & 3695 & 9.94 & 8 \\
\hline A30 & 1924.8 & 4612 & 14.75 & 13 \\
\hline B30 & 1925.43 & 4464 & 14.84 & 13 \\
\hline C30 & 1667.65 & 4356 & 13.26 & 15 \\
\hline A40 & 1924.8 & 4972 & 22.5 & 14 \\
\hline B40 & 1925.43 & 5353 & 18.83 & 16 \\
\hline C40 & 1907.8 & 4947 & 22.64 & 14 \\
\hline A50 & 1924.8 & 5748 & 27.39 & 21 \\
\hline B50 & 1925.43 & 6462 & 25.13 & 20 \\
\hline D50 & 1907.8 & 5422 & 24.65 & 25 \\
\hline
\end{tabular}

Table 1: Features of Test Problem Instances

Tables 2, 3, 4, and 5 report the computational results over the four sets of problem instances. The columns display, respectively, the name of the instance and its dimension, the number of samples created, their dimensions, the value $1-\epsilon$, the average of $H^{1-\epsilon}$ over the number of samples, the standard deviation, and the computing time in CPU seconds.

The experimental results indicate that increasing the dimension of the sample yields more precise solutions. The number of samples is relatively less important. The results also indicate that, in general, the standard deviations are very good, being average estimated values - standard deviation ratios smaller than $2 \%$ even in the worst case.

Regarding possible biases, we compared the values obtained by using our algorithm to those obtained by computing $H^{1-\epsilon}$ using a single sample of cardinality 100000. As supported by the figures in Table 6 , the values we found are very good.

To conclude, notice that the values of $H^{1-\epsilon}$ are remarkably smaller than those of the Hamiltonian path characterizing segments. They should actually be even better in practice. This is because in our experimentation we considered probabilities associated to optional stops uniformly distributed in the interval $(0,1)$. Yet, in the real word, stops with a value close to 1 would most likely be chosen as compulsory stops. In other words, in an actual implementation of a DAS service, optional stops are not requested frequently and consequently we expect better $H^{1-\epsilon}$ - Hamiltonian path-length ratios.

\section{Conclusions}

In this paper, we examined from a scheduling point of view a new type of semi-flexible transit service, the Demand-Adaptive Service. Comparing it to traditional transit and dial-a-ride services, we introduced the "new" scheduling requirements of DAS, which we identified as the 


\begin{tabular}{|c|c|c|c|c|c|c|}
\hline Name\&Dim. & nSamples & dimSamples & 1-epsilon & $H^{1-\epsilon}$ & Dev-Strd & Time (sec.) \\
\hline $\mathrm{A} 20$ & 10 & 50 & 0.95 & 3470.21 & 68.0074 & 3.46 \\
\hline A20 & 10 & 100 & 0.95 & 3472.21 & 60.9672 & 6.92 \\
\hline A20 & 10 & 150 & 0.95 & 3508.21 & 51.4296 & 10.38 \\
\hline A20 & 10 & 200 & 0.95 & 3504.21 & 39.2556 & 13.89 \\
\hline A20 & 10 & 250 & 0.95 & 3496.21 & 38.4318 & 17.34 \\
\hline A20 & 20 & 50 & 0.95 & 3476.21 & 81.2219 & 6.93 \\
\hline A20 & 20 & 100 & 0.95 & 3481.21 & 71.3583 & 13.87 \\
\hline A20 & 20 & 150 & 0.95 & 3497.21 & 55.9553 & 20.81 \\
\hline A20 & 20 & 200 & 0.95 & 3501.21 & 45.7384 & 27.72 \\
\hline A20 & 20 & 250 & 0.95 & 3491.21 & 36.4829 & 34.68 \\
\hline A20 & 30 & 50 & 0.95 & 3492.88 & 98.818 & 11.32 \\
\hline A20 & 30 & 100 & 0.95 & 3478.21 & 67.6535 & 22.62 \\
\hline $\mathrm{A} 20$ & 30 & 150 & 0.95 & 3490.88 & 56.1783 & 33.86 \\
\hline A20 & 30 & 200 & 0.95 & 3500.21 & 42.0714 & 45.21 \\
\hline A20 & 30 & 250 & 0.95 & 3492.88 & 34.3074 & 56.65 \\
\hline $\mathrm{B} 20$ & 10 & 50 & 0.95 & 3836.21 & 48.5283 & 3.63 \\
\hline $\mathrm{B} 20$ & 10 & 100 & 0.95 & 3840.21 & 38 & 7.22 \\
\hline B20 & 10 & 150 & 0.95 & 3822.21 & 31.241 & 10.81 \\
\hline $\mathrm{B} 20$ & 10 & 200 & 0.95 & 3830.21 & 30.7246 & 14.45 \\
\hline $\mathrm{B} 20$ & 10 & 250 & 0.95 & 3820.21 & 32.2955 & 18.03 \\
\hline B20 & 20 & 50 & 0.95 & 3827.21 & 48.2701 & 7.24 \\
\hline $\mathrm{B} 20$ & 20 & 100 & 0.95 & 3837.21 & 41.1461 & 14.45 \\
\hline $\mathrm{B} 20$ & 20 & 150 & 0.95 & 3822.21 & 31.8904 & 21.59 \\
\hline $\mathrm{B} 20$ & 20 & 200 & 0.95 & 3836.21 & 34.6121 & 28.82 \\
\hline$\overline{\mathrm{B} 20}$ & 20 & 250 & 0.95 & 3830.21 & 36.1386 & 36.01 \\
\hline $\mathrm{B} 20$ & 30 & 50 & 0.95 & 3824.88 & 49.7896 & 11.78 \\
\hline $\mathrm{B} 20$ & 30 & 100 & 0.95 & 3836.88 & 43.2088 & 23.53 \\
\hline $\mathrm{B} 20$ & 30 & 150 & 0.95 & 3833.55 & 36.4417 & 35.42 \\
\hline$\overline{\mathrm{B} 20}$ & 30 & 200 & 0.95 & 3836.88 & 37.6298 & 47.15 \\
\hline $\mathrm{B} 20$ & 30 & 250 & 0.95 & 3824.88 & 32.6803 & 58.82 \\
\hline $\mathrm{C} 20$ & 10 & 50 & 0.95 & 3124.21 & 42.2019 & 3.49 \\
\hline $\mathrm{C} 20$ & 10 & 100 & 0.95 & 3128.21 & 33.5708 & 6.99 \\
\hline $\mathrm{C} 20$ & 10 & 150 & 0.95 & 3112.21 & 10.8628 & 10.46 \\
\hline $\mathrm{C} 20$ & 10 & 200 & 0.95 & 3114.21 & 9.05539 & 13.94 \\
\hline $\mathrm{C} 20$ & 10 & 250 & 0.95 & 3116.21 & 10.8628 & 17.4 \\
\hline $\mathrm{C} 20$ & 20 & 50 & 0.95 & 3116.21 & 48.9183 & 6.99 \\
\hline $\mathrm{C} 20$ & 20 & 100 & 0.95 & 3129.21 & 34.5254 & 13.94 \\
\hline $\mathrm{C} 20$ & 20 & 150 & 0.95 & 3119.21 & 18.735 & 20.97 \\
\hline $\mathrm{C} 20$ & 20 & 200 & 0.95 & 3117.21 & 15.7162 & 27.93 \\
\hline $\mathrm{C} 20$ & 20 & 250 & 0.95 & 3112.21 & 13.8924 & 34.99 \\
\hline $\mathrm{C} 20$ & 30 & 50 & 0.95 & 3112.88 & 48.7237 & 11.46 \\
\hline $\mathrm{C} 20$ & 30 & 100 & 0.95 & 3134.88 & 32.4962 & 22.87 \\
\hline $\mathrm{C} 20$ & 30 & 150 & 0.95 & 3116.88 & 18.4662 & 34.26 \\
\hline $\mathrm{C} 20$ & 30 & 200 & 0.95 & 3114.21 & 16.3401 & 45.79 \\
\hline $\mathrm{C} 20$ & 30 & 250 & 0.95 & 3115.55 & 15.4596 & 57.28 \\
\hline
\end{tabular}

Table 2: Results for Segments with 20 Nodes 


\begin{tabular}{|c|c|c|c|c|c|c|}
\hline Name\&Dim. & nSamples & dimSamples & 1-epsilon & $H^{1-\epsilon}$ & Dev-Strd & Time (sec.) \\
\hline A30 & 10 & 50 & 0.95 & 3926.21 & 86.3423 & 4.11 \\
\hline A30 & 10 & 100 & 0.95 & 3924.21 & 58.8303 & 8.27 \\
\hline A30 & 10 & 150 & 0.95 & 3932.21 & 53.2635 & 12.37 \\
\hline A30 & 10 & 200 & 0.95 & 3936.21 & 42.3674 & 16.49 \\
\hline A30 & 10 & 250 & 0.95 & 3950.21 & 31.9844 & 20.6 \\
\hline A30 & 20 & 50 & 0.95 & 3938.21 & 76.2889 & 8.26 \\
\hline A30 & 20 & 100 & 0.95 & 3926.21 & 63.6946 & 16.45 \\
\hline A30 & 20 & 150 & 0.95 & 3938.21 & 49.6286 & 24.74 \\
\hline A30 & 20 & 200 & 0.95 & 3934.21 & 36.8511 & 32.92 \\
\hline A30 & 20 & 250 & 0.95 & 3937.21 & 31.1609 & 41.17 \\
\hline A30 & 30 & 50 & 0.95 & 3933.55 & 81.4187 & 12.4 \\
\hline A30 & 30 & 100 & 0.95 & 3926.21 & 57.7062 & 24.75 \\
\hline A30 & 30 & 150 & 0.95 & 3930.88 & 47.1381 & 37.09 \\
\hline A30 & 30 & 200 & 0.95 & 3929.55 & 40.6448 & 49.31 \\
\hline A30 & 30 & 250 & 0.95 & 3936.88 & 36.1525 & 61.61 \\
\hline B30 & 10 & 50 & 0.95 & 4062.21 & 50.7642 & 5.54 \\
\hline B30 & 10 & 100 & 0.95 & 4088.21 & 38.0132 & 11.13 \\
\hline B30 & 10 & 150 & 0.95 & 4096.21 & 38.4187 & 16.7 \\
\hline B30 & 10 & 200 & 0.95 & 4088.21 & 32.2645 & 22 \\
\hline B30 & 10 & 250 & 0.95 & 4102.21 & 25.5734 & 27.6 \\
\hline B30 & 20 & 50 & 0.95 & 4075.21 & 52.7257 & 11.15 \\
\hline B30 & 20 & 100 & 0.95 & 4102.21 & 41.7133 & 21.99 \\
\hline B30 & 20 & 150 & 0.95 & 4104.21 & 34.9571 & 33.37 \\
\hline B30 & 20 & 150 & 0.95 & 4104.21 & 34.9571 & 33.37 \\
\hline B30 & 20 & 200 & 0.95 & 4089.21 & 40.4475 & 44.03 \\
\hline B30 & 20 & 250 & 0.95 & 4095.21 & 32.5883 & 54.95 \\
\hline B30 & 30 & 50 & 0.95 & 4086.21 & 59.0931 & 16.73 \\
\hline B30 & 30 & 100 & 0.95 & 4109.55 & 43.8634 & 33.32 \\
\hline B30 & 30 & 150 & 0.95 & 4096.88 & 37.2827 & 49.67 \\
\hline B30 & 30 & 200 & 0.95 & 4084.21 & 40.3733 & 65.4 \\
\hline B30 & 30 & 250 & 0.95 & 4098.21 & 28.7054 & 81.89 \\
\hline C30 & 10 & 50 & 0.95 & 3362.21 & 20.4695 & 3.8 \\
\hline C30 & 10 & 100 & 0.95 & 3390.21 & 33.2716 & 7.51 \\
\hline C30 & 10 & 150 & 0.95 & 3372.21 & 27.55 & 11.22 \\
\hline C30 & 10 & 200 & 0.95 & 3358.21 & 28.0535 & 14.99 \\
\hline C30 & 10 & 250 & 0.95 & 3366.21 & 24.0208 & 18.7 \\
\hline C30 & 20 & 50 & 0.95 & 3375.21 & 41.1825 & 7.56 \\
\hline C30 & 20 & 100 & 0.95 & 3370.21 & 32.573 & 14.98 \\
\hline C30 & 20 & 150 & 0.95 & 3367.21 & 29.7825 & 22.41 \\
\hline C30 & 20 & 200 & 0.95 & 3356.21 & 26.7208 & 29.8 \\
\hline C30 & 20 & 250 & 0.95 & 3362.21 & 23.9374 & 37.22 \\
\hline C30 & 30 & 50 & 0.95 & 3368.21 & 42.0357 & 11.26 \\
\hline C30 & 30 & 100 & 0.95 & 3373.55 & 32.1092 & 22.37 \\
\hline C30 & 30 & 150 & 0.95 & 3363.55 & 32.5883 & 33.54 \\
\hline C30 & 30 & 200 & 0.95 & 3354.21 & 27.8747 & 44.71 \\
\hline C30 & 30 & 250 & 0.95 & 3363.55 & 22.4277 & 55.78 \\
\hline
\end{tabular}

Table 3: Results for Segments with 30 Nodes 


\begin{tabular}{|c|c|c|c|c|c|c|}
\hline Name\&Dim. & nSamples & dimSamples & 1-epsilon & $H^{1-\epsilon}$ & Dev-Strd & Time (sec.) \\
\hline A40 & 10 & 50 & 0.95 & 4552.21 & 52.4595 & 7.46 \\
\hline A40 & 10 & 100 & 0.95 & 4568.21 & 30.9516 & 14.67 \\
\hline A40 & 10 & 150 & 0.95 & 4562.21 & 33.6452 & 21.88 \\
\hline $\mathrm{A} 40$ & 10 & 200 & 0.95 & 4564.21 & 22.2711 & 29.4 \\
\hline A40 & 10 & 250 & 0.95 & 4562.21 & 22.1811 & 36.67 \\
\hline A40 & 20 & 50 & 0.95 & 4554.21 & 46.9042 & 14.68 \\
\hline A40 & 20 & 100 & 0.95 & 4567.21 & 29.189 & 29.4 \\
\hline A40 & 20 & 150 & 0.95 & 4557.21 & 27.0555 & 44.17 \\
\hline $\mathrm{A} 40$ & 20 & 200 & 0.95 & 4564.21 & 24.0832 & 58.71 \\
\hline A40 & 20 & 250 & 0.95 & 4560.21 & 20.1494 & 73.81 \\
\hline A40 & 30 & 50 & 0.95 & 4557.55 & 51.9808 & 21.86 \\
\hline A40 & 30 & 100 & 0.95 & 4564.21 & 29.2575 & 44.16 \\
\hline A40 & 30 & 150 & 0.95 & 4558.88 & 27.7849 & 66.37 \\
\hline A40 & 30 & 200 & 0.95 & 4562.21 & 22.4944 & 88.1 \\
\hline A40 & 30 & 250 & 0.95 & 4557.55 & 20.9284 & 109.74 \\
\hline B40 & 10 & 50 & 0.95 & 4362.21 & 23.9165 & 34.8 \\
\hline $\mathrm{B} 40$ & 10 & 100 & 0.95 & 4366.21 & 23.9165 & 64.79 \\
\hline B40 & 10 & 150 & 0.95 & 4378.21 & 11.8322 & 98.96 \\
\hline B40 & 10 & 200 & 0.95 & 4376.21 & 24.3311 & 131.69 \\
\hline B40 & 10 & 250 & 0.95 & 4382.21 & 20.2485 & 166.35 \\
\hline B40 & 20 & 50 & 0.95 & 4373.21 & 34.322 & 64.82 \\
\hline B40 & 20 & 100 & 0.95 & 4369.21 & 31.5595 & 131.72 \\
\hline B40 & 20 & 150 & 0.95 & 4382.21 & 21.4009 & 197.53 \\
\hline B40 & 20 & 200 & 0.95 & 4373.21 & 20.4939 & 261.99 \\
\hline B40 & 20 & 250 & 0.95 & 4375.21 & 24.0832 & 322.11 \\
\hline B40 & 30 & 50 & 0.95 & 4375.55 & 38.3406 & 98.98 \\
\hline B40 & 30 & 100 & 0.95 & 4370.21 & 31.3369 & 197.57 \\
\hline B40 & 30 & 150 & 0.95 & 4373.55 & 22.9783 & 287.2 \\
\hline B40 & 30 & 200 & 0.95 & 4370.21 & 22.4054 & 388.42 \\
\hline $\mathrm{C} 40$ & 10 & 50 & 0.95 & 4258.21 & 77.3434 & 10.67 \\
\hline $\mathrm{C} 40$ & 10 & 100 & 0.95 & 4256.21 & 53.963 & 20.65 \\
\hline $\mathrm{C} 40$ & 10 & 150 & 0.95 & 4284.21 & 51.5364 & 30.84 \\
\hline $\mathrm{C} 40$ & 10 & 200 & 0.95 & 4314.21 & 46.4327 & 42.43 \\
\hline $\mathrm{C} 40$ & 10 & 250 & 0.95 & 4314.21 & 49.7594 & 52.17 \\
\hline $\mathrm{C} 40$ & 20 & 50 & 0.95 & 4256.21 & 81.4739 & 20.63 \\
\hline $\mathrm{C} 40$ & 20 & 100 & 0.95 & 4293.21 & 62.1289 & 42.4 \\
\hline $\mathrm{C} 40$ & 20 & 150 & 0.95 & 4312.21 & 59.3127 & 62.74 \\
\hline $\mathrm{C} 40$ & 20 & 200 & 0.95 & 4323.21 & 37.6829 & 83.53 \\
\hline $\mathrm{C} 40$ & 20 & 250 & 0.95 & 4323.21 & 44.4747 & 104.5 \\
\hline $\mathrm{C} 40$ & 30 & 50 & 0.95 & 4273.55 & 86.406 & 30.82 \\
\hline $\mathrm{C} 40$ & 30 & 100 & 0.95 & 4294.88 & 61.2862 & 62.75 \\
\hline $\mathrm{C} 40$ & 30 & 150 & 0.95 & 4316.21 & 60.1498 & 95.12 \\
\hline $\mathrm{C} 40$ & 30 & 200 & 0.95 & 4324.88 & 43.2666 & 123.76 \\
\hline $\mathrm{C} 40$ & 30 & 250 & 0.95 & 4318.88 & 45.5851 & 153.86 \\
\hline
\end{tabular}

Table 4: Results for Segments with 40 Nodes 


\begin{tabular}{|c|c|c|c|c|c|c|}
\hline Name\&Dim. & nSamples & dimSamples & 1-epsilon & $H^{1-\epsilon}$ & Dev-Strd & time (sec.) \\
\hline A50 & 10 & 50 & 0.95 & 4960.21 & 43.795 & 33.38 \\
\hline A50 & 10 & 100 & 0.95 & 4954.21 & 26.7582 & 63.34 \\
\hline A50 & 10 & 150 & 0.95 & 4964.21 & 29.9333 & 97.89 \\
\hline A50 & 10 & 200 & 0.95 & 4970.21 & 34.3802 & 128.29 \\
\hline A50 & 10 & 250 & 0.95 & 4968.21 & 19.9499 & 162.8 \\
\hline$\overline{\mathrm{A} 50}$ & 20 & 50 & 0.95 & 4962.21 & 39.7744 & 63.34 \\
\hline A50 & 20 & 100 & 0.95 & 4962.21 & 40.2492 & 128.26 \\
\hline A50 & 20 & 150 & 0.95 & 4969.21 & 30.9839 & 191.93 \\
\hline A50 & 20 & 200 & 0.95 & 4963.21 & 32.619 & 252.65 \\
\hline A50 & 20 & 250 & 0.95 & 4953.21 & 32.0312 & 308.64 \\
\hline A50 & 30 & 50 & 0.95 & 4970.21 & 47.7703 & 97.9 \\
\hline A50 & 30 & 100 & 0.95 & 4954.21 & 39.3954 & 191.93 \\
\hline A50 & 30 & 150 & 0.95 & 4958.88 & 37.2022 & 278.73 \\
\hline A50 & 30 & 200 & 0.95 & 4958.88 & 31.3688 & 365.49 \\
\hline A50 & 30 & 250 & 0.95 & 4950.21 & 30.0333 & 453.32 \\
\hline B50 & 10 & 50 & 0.95 & 4854.21 & 33.3766 & 41.39 \\
\hline B50 & 10 & 100 & 0.95 & 4880.21 & 45.607 & 83.79 \\
\hline B50 & 10 & 150 & 0.95 & 4874.21 & 21.7256 & 125.1 \\
\hline B50 & 10 & 200 & 0.95 & 4874.21 & 23.5372 & 174.17 \\
\hline B50 & 10 & 250 & 0.95 & 4884.21 & 22.2261 & 214.45 \\
\hline B50 & 20 & 50 & 0.95 & 4871.21 & 47.5184 & 83.8 \\
\hline $\bar{B} 50$ & 20 & 100 & 0.95 & 4887.21 & 40.2492 & 174.18 \\
\hline B50 & 20 & 150 & 0.95 & 4881.21 & 23.1517 & 255.68 \\
\hline B50 & 20 & 200 & 0.95 & 4888.21 & 32.3419 & 340.3 \\
\hline B50 & 20 & 250 & 0.95 & 4888.21 & 29.0861 & 430.86 \\
\hline B50 & 30 & 50 & 0.95 & 4870.88 & 45.9565 & 125.09 \\
\hline B50 & 30 & 100 & 0.95 & 4890.21 & $\begin{array}{l}37.1483 \\
\end{array}$ & 255.78 \\
\hline B50 & 30 & 150 & 0.95 & 4888.21 & 28.9482 & 387.66 \\
\hline B50 & 30 & 200 & 0.95 & 4886.88 & 28.4956 & 513.3 \\
\hline B50 & 30 & 250 & 0.95 & 4888.88 & 28.2489 & 638.01 \\
\hline $\mathrm{C} 50$ & 10 & 50 & 0.95 & 4496.21 & 43.2435 & 88.62 \\
\hline C50 & 10 & 100 & 0.95 & 4518.21 & 51.1859 & 184.92 \\
\hline C50 & 10 & 150 & 0.95 & 4506.21 & 41.1339 & 273.92 \\
\hline $\mathrm{C} 50$ & 10 & 200 & 0.95 & 4494.21 & 21.8174 & 363.81 \\
\hline $\mathrm{C} 50$ & 10 & 250 & 0.95 & 4500.21 & 23.622 & 436.01 \\
\hline $\mathrm{C} 50$ & 20 & 50 & 0.95 & 4511.21 & 55.9464 & 184.86 \\
\hline $\mathrm{C} 50$ & 20 & 100 & 0.95 & 4515.21 & 45.3652 & 364.03 \\
\hline $\mathrm{C} 50$ & 20 & 100 & 0.95 & 4515.21 & 45.3652 & 364.03 \\
\hline $\mathrm{C} 50$ & 20 & 150 & 0.95 & 4497.21 & 41.6173 & 521.95 \\
\hline $\mathrm{C} 50$ & 20 & 200 & 0.95 & 4487.21 & 23.0651 & 686.62 \\
\hline $\mathrm{C} 50$ & 20 & 250 & 0.95 & 4495.21 & 30.5941 & 851.22 \\
\hline $\mathrm{C} 50$ & 30 & 50 & 0.95 & 4508.21 & 50.9706 & 273.88 \\
\hline C50 & 30 & 100 & 0.95 & 4514.21 & 53.7215 & 521.82 \\
\hline C50 & 30 & 150 & 0.95 & 4491.55 & 38.9615 & 766.99 \\
\hline $\mathrm{C} 50$ & 30 & 200 & 0.95 & 4486.88 & 20.3961 & 1016.13 \\
\hline C50 & 30 & 250 & 0.95 & 4490.21 & 28.5657 & 1265.88 \\
\hline
\end{tabular}

Table 5: Results for Segments with 50 Nodes

\begin{tabular}{|c|c|c|c|c|c|c|}
\hline Name\&Dim. & nSamples & dimSamples & 1-epsilon & $H^{1-\epsilon}$ & Dev-Strd & Time sec. \\
\hline A20 & 1 & 100000 & 0.95 & 3514.21 & 0 & 714.33 \\
\hline A30 & 1 & 100000 & 0.95 & 3954.21 & 0 & 848.39 \\
\hline A40 & 1 & 100000 & 0.95 & 4554.21 & 0 & 1472.54 \\
\hline A50 & 1 & 100000 & 0.95 & 4954.21 & 0 & 5983.55 \\
\hline
\end{tabular}

Table 6: Values of $H^{1-\epsilon}$ Computed over 100000-large Samples 
construction of a master schedule. We formalized the master scheduling problem for a single DAS line and proposed a solution framework based on decoupling the origin-destination demand and using a particular sampling technique. Computational results show that the method we propose is efficient and produces high-quality results.

\section{Acknowledgments}

Partial funding for this research was provided by Natural Sciences and Engineering Research Council of Canada (NSERC) through its Industrial Research Chair and Discovery grant programs. This research was also partially funded by Regione Lombardia, Italy, through its INGENIO grants.

Fausto Errico wishes to thank Professor Stein W. Wallace for an enlightening conversation held in Montreal in December 2006.

While working on this project, Dr. Teodor Gabriel Crainic was Adjunct Professor at the Department of Computer Science and Operations Research of the Université de Montréal, Canada, and at Molde University College, Norway.

\section{References}

[1] Carpaneto, G., Dell'Amico, M., and Toth, P. Exact Solution of Large-scale, Asymmetric Traveling Salesman Problems. ACM Trans. Math. Softw., 21(4):394-409, 1995.

[2] Ceder, A. and Wilson, H.M. Public Transport Operations Planning. In Design and Operation of Civil and Environmental Engineering systems, pages 395-434. John Wiley \& Sons, Inc., New York, 1997.

[3] Cordeau, J.-F. and Laporte, G. The Dial-a-Ride Problem (DARP): Variants, Modeling Issues and Algorithms. 4OR, 1(2):89-101, 2003.

[4] Crainic, T.G., Malucelli, F, and Nonato, M. A Demand Responsive Feeder Bus System. In CD-ROM of 7th World Congress on Intelligent Transport Systems. 7WC-ITS, Torino, Italia, 2000.

[5] Crainic, T.G., Malucelli, F, and Nonato, M. Flexible Many-to-few + Few-to-many $=$ An Almost Personalized Transit System. In Preprints TRISTAN IV - Triennial Symposium on Transportation Analysis, volume 2, pages 435-440. Faculdade de Ciências da Universidade de Lisboa and Universidade dos Açores, São Miguel, Açores, Portugal, 2001. 
[6] Crainic, T.G., Malucelli, F, Nonato, M., and Guertin, F. Meta-Heuristics for a Class of Demand-Responsive Transit Systems. INFORMS Journal on Computing, 17(1):10-24, 2005.

[7] Errico, F. The design of flexible transit systems: models and solution methods. PhD thesis, Politecnico di Milano, Italy, 2008.

[8] Ioachim, I., Desrosiers, J., Dumas, Y., and Solomon, M.M. A Request Clustering for Door to Door Handicapped Transportation. Transportation Science, 29(1):63-68, 1995.

[9] Malucelli, F, Nonato, M., Crainic, T.G., and Guertin, F. Adaptive Memory Programming for a Class of Demand-Responsive Transit Systems. In Voß, S. and Daduna, J.R., editors, Computer-Aided Scheduling of Public Transport, volume 505 of Lecture Notes in Economics and Mathematical Systems, pages 253-273. Springer, Berlin, 2001.

[10] Malucelli, F, Nonato, M., and Pallottino, S. Some Proposals on Flexible Transit. In Ciriani, T.A., Johnson, E.L., and Tadei, R., editors, Operations Research in Industry, pages 157-182. McMillian, 1999.

[11] Quadrifoglio, L., Dessouky, M.M., and Palmer, K. An Insertion Heuristic for Scheduling Mobility Allowance Shuttle Transit (MAST) Services. Journal of Scheduling, 10(1):2540, 2007.

[12] Toth, P. and Vigo, D. Fast Local Search Algorithms for the Handicapped Persons Transportation Problem. In Osman, I.H. and Kelly, J.P., editors, Meta-Heuristics: Theory \& Applications, pages 677-690. Kluwer Academic Publishers, Norwell, MA, 1996. 